\title{
UAV-Based Physical-Layer Intelligent Technologies for 5G-Enabled Internet of Things: A Survey
}

\author{
Changyu Wang, ${ }^{1}$ Weili Yu, ${ }^{1}$ Jinrong Lu $\left(\mathbb{D},{ }^{2,3}\right.$ Fusheng Zhu $\left(\mathbb{D},{ }^{4}\right.$ Lihua Fan, ${ }^{5}$ and Shengping Li ${ }^{6}$ \\ ${ }^{1}$ Air Force Aviation University, Changchun, Jilin, China \\ ${ }^{2}$ School of Computer Science, Guangzhou University, Guangzhou, China \\ ${ }^{3}$ Guangdong Provincial Key Laboratory of Building Energy Efficiency and Application Technologies, Guangzhou University, \\ Guangzhou, China \\ ${ }^{4}$ Guangdong New Generation Communication and Network Innovative Institute (GDCNi), Guangzhou, China \\ ${ }^{5}$ School of Electronic Information, Shunde Polytechnic, Foshan, Guangdong, China \\ ${ }^{6}$ College of Engineering, Shantou University, Shantou, Guangdong, China
}

Correspondence should be addressed to Fusheng Zhu; zhufusheng@gdcni.cn

Received 8 December 2021; Revised 4 January 2022; Accepted 10 January 2022; Published 28 January 2022

Academic Editor: Xingwang Li

Copyright (c) 2022 Changyu Wang et al. This is an open access article distributed under the Creative Commons Attribution License, which permits unrestricted use, distribution, and reproduction in any medium, provided the original work is properly cited.

\begin{abstract}
In recent years, the utilization and application of unmanned aerial vehicle (UAV) have attracted much attention, both from academy and industry. UAVs have been widely used in many practical communication scenarios, due to its high flexibility, high mobility, and low cost. Therefore, this paper addresses the key technologies of UAV communication and reviews the current research status, from various aspects including UAV communication transmission, UAV formation control and networking, UAV resource allocation, and intelligent communication from artificial intelligence algorithms. Then, artificial intelligence is introduced into multiple aspects of UAV communication, including channel transmission, control and networking, and resource scheduling, to organically integrate artificial intelligence into UAV communication, which can help reduce the complexity of communication algorithms and improve system spectrum efficiency. This paper will help improve the efficiency of UAV communication and promote the development of UAV-related industries.
\end{abstract}

\section{Research Background}

Unmanned aerial vehicle (UAV) is a new and evolving technology that has gained much attention over the past few decades due to its flexibility, high speed mobility, and low cost [1-3]. Initially, UAVs were mainly used in military operations to perform critical missions and reduce pilot damage. But as the cost of manufacturing UAVs have continued to decrease and the size of the equipment has been miniaturized, numerous other applications for UAVs have emerged [4-6]. As shown in Figure 1, typical UAV applications include emergency search and rescue, climate monitoring, traffic control, forest fire detection, cargo logistics, aerial photography, and communications.

Motivated by the development and deployment of the fifth-generation (5G) communication [7-9], UAV communication has been successfully applied in the academy and industry, due to the advantages of flexible deployment, wide coverage, and no regional restrictions. Many countries around the world have carried out research on UAV communication. In 2016, the Central Committee of the Communist Party of China, the State Council and the Central Military Commission issued the Opinions on the Integrated Development of Economic Construction and National Defence Construction, pointing out that UAV communication is an important part of the integration of military and civilian construction of air, space, and sky networks. In 2017, the State Council issued the Development Plan for a New Generation of Artificial Intelligence, which called for the construction of an innovative theoretical system for the autonomous collaborative control and optimal decision of intelligent 


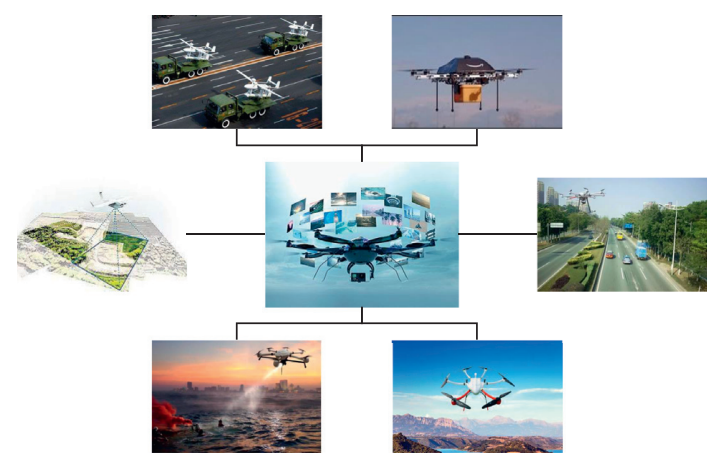

FIgURE 1: Typical application diagram of UAV.

unmanned systems. In March 2020, in order to implement the overall plan of the State Council's New Generation Artificial Intelligence Development Plan, the Ministry of Science and Technology further launched the implementation of the New Generation Artificial Intelligence Major Science and Technology Project Implementation Plan.

In order to achieve efficient UAV swarm communication coverage, smooth communication links need to be maintained at all times between swarm UAVs and UAVs or between UAVs and service terminals. The future UAV communication network needs to support a user experience rate of gigabits per second, fast and flexible UAV swarm networking, low latency control signaling, and 100 megabit system bandwidth. Considering the demand for high-capacity and high-rate communications for swarm communications, the technological focus of UAV communications is beginning to shift to the high frequency band such as millimeter wave $(30-300 \mathrm{GHz})$. Millimeter waves can provide larger bandwidth resources, enabling user rates up to gigabits per second. At the same time, the tiny millimeter wave wavelength allows hundreds of antennas to be mounted in a small space or integrated into a chip, giving UAVs ultra-high-resolution beam assignment capabilities. Therefore, with the help of millimeter wave and multiantenna technology $[10,11]$, UAV swarm communication system is expected to realize broadband wireless transmission through collaboration and networking and improve network capacity and link reliability through multi-UAV formation, rapid deployment, and collaboration. In addition to the aforementioned techniques, some other techniques, such as the reconfigurable intelligent surfaces [12-17] and multiple access [18-20], can be integrated to the UAV communication systems, in order to further enhance the system performance.

However, UAV swarm communication based on millimeter-wave and multiple antennas also faces great challenges. The sparse characteristics of millimeter-wave propagation in high frequency bands, the application of multiple antennas, the high-speed mobility of UAVs, the dynamic change of formations, and the limited multidimensional resources of swarms cause that the key technologies of swarm communication need to be reexamined. UAV swarm communication breaks the application scope of traditional mobile communication systems. The unified technical framework which supports the extremely different and complicated applications of UAV swarm faces unprecedented challenges.

In recent years, artificial intelligence techniques [21-23] have continued to evolve with the rapid development of intelligent mobile computing and data storage technologies [24-26]. Artificial intelligence can find its typical applications for many scenarios including statistics, inference, fitting, optimization, and clustering of data. The rapid development of artificial intelligence techniques offers potential possibilities to address the challenges of swarm communication and to surpass design concepts and performance of the traditional mobile communication. This paper will design a swarm intelligent communication transmission strategy to break the traditional communication model and introduce artificial intelligence into multiple levels of UAV swarm communication, such as broadband transmission, formation control and networking, and multidimensional resource allocation, to substantially improve the performance of the swarm communication system, realize the organic integration of artificial intelligence and UAV swarm communication system, and promote the development and maturity of UAV swarm communication.

\section{Research Progress and Challenges of UAV Swarm Communication Transmission}

With the rapid development of automatic control and artificial intelligence technology, UAV communication is widely used in various fields such as aerial photography, logistics, and entertainment, which has attracted much attention from researches and engineers at home and abroad. For swarm communication, the design and performance evaluation of the system require the establishment of UAV communication channel models. In general, the channel model can be divided into two categories: deterministic channel models and random channel model. Unlike the deterministic model, the stochastic model has high accuracy and low complexity, and it can be more easily extended to various wireless communication application scenarios. In this direction, a three-dimensional geometric stochastic model could be proposed to describe the communication channel, which assumed that mobile transmitter and receiver moved in a flat environment and did not consider the effect of ground reflection on the channel modeling. A 3D ellipsoidal channel model could be designed for high-altitude platforms, which did not consider the effect of platform elevation angle on the channel modeling. The technique of multiple-input multiple-output could be integrated into the high-altitude platform system and proposed a three-dimensional geometric single-hop reflection reference model. At the same time, different from traditional terrestrial cellular mobile communication networks, millimeter wave wavelengths are shorter and have severe path loss, and millimeter wave-based UAV broadband communication links are highly susceptible to atmospheric environment, terrain, and various obstacles. The beamforming technique could be applied to improve the signal quality at the UAV 
receiver. Moreover, single- and multilayer beamforming methods could be utilized for large-scale antenna arrays. Furthermore, a simulated beam assignment method could be provided for a single RF link to multiple antenna channels. However, the existing works lack a comprehensive consideration of the number and cost of RF links and the performance of analog and digital beamforming algorithms. It is hence urgent to study the intelligent communication strategies to support UAV swarms in complied application scenarios, including channel modeling, channel information acquisition, and hybrid beam assignment and tracking.

\section{Research Progress and Challenges of UAV Swarm Communication Formation Control and Networking}

Formation control is the key technology of UAV swarm cooperative communication and networking. In the direction, a formation strategy was proposed based on a star network structure to ensure UAV network routing performance. A multihop reactive AODV routing protocol was devised to adapt to the change in dynamic wireless links. However, the protocol search time was long when the link was unstable, which could not meet the high dynamic communication requirements of UAVs. A UAV formation strategy could be designed by aiming at energy and time optimization. The use of UAVs could be used as the network structure of aerial base stations, where we could analyze the relationship between UAV height and ground coverage and obtain the UAV height under the best ground coverage of UAV. The relationship between the number of drone hovering times and the coverage probability of the ground terminal could be obtained through theoretical analysis. A spatial three-dimensional Poisson point process model could be utilized to obtain the communication interruption probability under different UAV densities and navigation altitudes of the base station deployment. Moreover, a wireless network relaying model for UAVs could be deployed to solve the problem of blind area coverage overlap. Furthermore, the multilayer core network of UAV based on millimeter-wave could be proposed. According to above literature review, we can find that there is lack of research on drone swarm control and collaborative networking in the existing works. Moreover, the UAV motion model based on the fixed route cannot fully characterize the dynamic and random change of the UAV path, and the existing routing method is limited by the communication range of the star network node, which cannot meet the requirement of large and dynamic UAV swarm formation activities. Therefore, it is urgent to study the UAV swarm communication control and networking technology suitable for UAV swarm communication characteristics and dynamic topology change.

\section{Research Progress and Challenges of UAV Swarm Communication Resource Allocation}

Due to the limited resources available for drone swarms in air navigation, the key to improving the communication performance in drone swarms is the allocation of various resources of drone swarms timely and efficiently. In the direction, the opportunities and challenges of millimeter-wave massive MIMO could be reviewed in 5G hotspot coverage, dense networking, and heterogeneous networking. The lower bound of the spectrum efficiency of massive MIMO systems could be analyzed, and the energy efficiency of distributed massive MIMO systems could be optimized through antenna selection, user clustering, and power control. A power consumption model could be established to optimize the number of base station antennas and improve the system energy efficiency. A joint carrier selection and resource allocation method could be devised based on a greedy algorithm to improve system throughput. Aiming at the problem of the limited supply of UAV platforms, an energy consumption optimization algorithm could be used for multi-UAV cooperative assistance ground communication. Aiming at different service quality requirements, random user scheduling strategies could be optimized based on the service and lacking analysis and developed multiple differentiated service types for future drone swarm communication. Considering the high mobility, dynamic topology, energy limitation, and communication link performance of drone swarm communication, it is urgent to study the resource allocation method that takes into account the multicategory business requirement and resource limitation of drones, which can help obtain the optimal system performance under limited resources, solve the bottleneck problem of drone swarm scheduling, and achieve reliable information transmission.

\section{Research Progress and Challenges of Intelligent Communication}

AI is a new technique that studies and exploits theories, methods, technologies, and application systems for simulating, extending, and expanding human intelligence. Intelligent communication methods jointly designed by AI and communications are expected to significantly improve the performance of communication systems [27, 28]. In this area, the authors in [29-31] proposed to utilize the federated learning to wireless communications, which opens a new window for the design of UAV-based communication systems. Moreover, some works based on the reinforcement learning in mobile edge computing networks $[32,33]$ could be applied to the UAV communication systems, in order to achieve a fine scheduling and trajectory design of multiple UAVs, which utilized Q-learning to improve the scheduling performance of the system. Furthermore, deep reinforcement learning and federation learning could be combined with edge computing in a joint design to improve the performance of mobile communication systems. A channel prediction method for large-scale MIMO communication systems could be proposed based on deep learning, which effectively reduced the complexity of downlink channel information acquisition and training feedback overhead. A machine learning-based hybrid precoding method for UAV millimeter-wave large-scale MIMO communication systems was proposed to improve the robustness of UAV communication transmission. However, the above existing works combining AI and communication mainly consider traditionally fixed base station scenarios and 
lack consideration of mobility, formation topology, and resource constraint of swarm UAV communication systems. Hence, it is urgent to study the use of advanced AI techniques to solve UAV swarm communication challenges.

It is clear from the above wide literature review that the existing works lack the study that integrates intelligent and efficient UAV swarm communication, formation control and collaborative networking, and resource allocation, and it is urgent to study communication, control, and networking methods that are applicable to the characteristics of swarms and dynamic topological change. Therefore, this paper introduces AI into multiple levels of UAV communication, including channel transmission, control and networking, and resource scheduling, to integrate AI with UAV swarm communication, thereby reducing the complexity of swarm communication algorithms and improving system spectrum efficiency. To address the challenges of swarm network navigation trajectory, attitude, formation topology, and large-scale dynamic change in space and time through efficient UAV swarm intelligent collaborative control and networking, we take into account the system spectrum efficiency, energy efficiency, user transmission rate, quality of service, and fairness and other requirements. We, in future, use AI to optimally schedule UAV swarm antenna, position, motion, attitude, carrier, spectrum, power, beam, storage, computation, and other resources, in order to achieve an efficient, robust, and intelligent resource allocation for the UAV swarm communication.

\section{Conclusions}

With its flexibility, high maneuverability, and low cost, UAV communication has promoted the application of UAV communication in emergency search and rescue, climate monitoring, traffic control, forest fire detection, cargo logistics, aerial photography, and other fields. Based on the key technologies of UAV communication, this paper summarized the current research status of UAV swarm communication transmission, UAV swarm communication formation control and networking, UAV swarm communication resource allocation, and intelligent communication and so on. Furthermore, AI was introduced into multiple aspects of UAV communication, including channel transmission, control and networking, and resource scheduling. In this work, AI was integrated into the UAV swarm communication to reduce the complexity of swarm communication algorithms and improve the system spectrum efficiency. The research in this paper will improve the efficiency of UAV communication and promote the development of UAV-related industries.

\section{Data Availability}

The data used to support the findings of this study are included in the article.

\section{Conflicts of Interest}

The authors declare that they have no conflicts of interest.

\section{Acknowledgments}

This work was supported by the Key-Area Research and Development Program of Guangdong Province, China (no. 2018B010124001), National Natural Science Foundation of China (no. 61573233), Team Project of the University of Guangdong Province (no. 2015KCXTD018), Key Project of Natural Science Foundation of Guangdong Province (no. 2015A030311017), research program of Guangzhou University (no. YJ2021003), and International Science and Technology Cooperation Projects of Guangdong Province (no. 2020A0505100060).

\section{References}

[1] Z. Na, J. Wang, C. Liu, M. Guan, and Z. Gao, "Join trajectory optimization and communication design for uav-enabled ofdm networks," Ad Hoc Networks, vol. 98, Article ID 102031, 2020.

[2] J. Liu, F. Gao, W. Jia, and W. Zhang, "Gridless compressed sensing based channel estimation for UAV wideband communications with beam squint," IEEE Transactions on Vehicular Technology, vol. 70, no. 10, pp. 10265-10277, 2021.

[3] L. Chen, "Intelligent ubiquitous computing for future UAVenabled MEC network systems," Cluster Computing, vol. 2021, no. 25, pp. 1-10, 2021.

[4] Y. Liu, J. Shi, C. Liu, and Z. Gao, "UAV-supported clustered NOMA for 6G-enabled internet of things: trajectory planning and resource allocation," IEEE Internet of Things Journal, vol. 8, no. 20, 2020.

[5] D. Deng, X. Li, V. Menon, M. J. Piran, H. Chen, and M. A. Jan, "Learning-based joint UAV trajectory and power allocation optimization for secure IoT networks," Digital Communications and Networks, vol. 54, pp. 2352-2361, 2021.

[6] J. Liu, J. Jiang, and F. Gao, "Efficient deployment with geometric analysis for mmwave UAV communications," IEEE Wireless Communications Letters, vol. 9, no. 7, pp. 1115-1119, 2020.

[7] S. Ni, "Multiband cooperation for $5 \mathrm{~g}$ hetnets: a promising network paradigm," IEEE Vehicular Technology Magazine, vol. 14, no. 4, pp. 85-93, 2019.

[8] N. Yang, L. Wang, G. Geraci, M. Elkashlan, J. Yuan, and M. Di Renzo, "Safeguarding $5 \mathrm{~g}$ wireless communication networks using physical layer security," IEEE Communications Magazine, vol. 53, no. 4, pp. 20-27, 2015.

[9] X. Sun, Q. Li, and X. Ma, "Edge caching and computation management for real-time internet of vehicles: an online and distributed approach," IEEE Transactions on Intelligent Transportation Systems, vol. 22, no. 4, pp. 2183-2197, 2021.

[10] B. Wang, F. Gao, S. Jin, H. Lin, and G. Y. Li, "Spatial-and frequency-wideband effects in millimeter-wave massive MIMO systems," IEEE Transactions on Signal Processing, vol. 66, no. 13, pp. 3393-3406, 2018.

[11] H. Xie, F. Gao, S. Zhang, and S. Jin, "A unified transmission strategy for TDD/FDD massive MIMO systems with spatial basis expansion model," IEEE Transactions on Vehicular Technology, vol. 66, no. 4, pp. 3170-3184, 2017.

[12] X. Hu, C. Zhong, Y. Zhu, X. Chen, and Z. Zhang, "Programmable metasurface-based multicast systems: design and analysis," IEEE Journal on Selected Areas in Communications, vol. 38, no. 8, pp. 1763-1776, 2020.

[13] G. Li, H. Liu, G. Huang, X. Li, B. Raj, and F. Kara, "Effective capacity analysis of reconfigurable intelligent surfaces aided 
NOMA network," EURASIP Journal on Wireless Communications and Networking, vol. 2021, no. 1, p. 198, 2021.

[14] Q. Tao, J. Wang, and C. Zhong, "Performance analysis of intelligent reflecting surface aided communication systems," IEEE Communications Letters, vol. 24, no. 11, pp. 2464-2468, 2020.

[15] X. Hu, C. Zhong, Y. Zhang, X. Chen, and Z. Zhang, "Location information aided multiple intelligent reflecting surface systems," IEEE Transactions on Communications, vol. 68, no. 12, pp. 7948-7962, 2020.

[16] X. Hu, J. Wang, and C. Zhong, "Statistical CSI based design for intelligent reflecting surface assisted MISO systems," Science China Information Sciences, vol. 63, no. 12, Article ID 222303, 2020.

[17] J. Zhang, Y. Zhang, C. Zhong, and Z. Zhang, "Robust design for intelligent reflecting surfaces assisted MISO systems," IEEE Communications Letters, vol. 24, no. 10, pp. 2353-2357, 2020.

[18] X. Li, M. Zhao, M. Zeng et al., "Hardware impaired ambient backscatter NOMA systems: reliability and security," IEEE Transactions on Communications, vol. 69, no. 4, pp. 27232736, 2021.

[19] Y. Zheng, W. U. Khan, M. Zeng, D. Li, G. K. Ragesh, and L. Li, "Physical layer security of cognitive ambient backscatter communications for green internet-of-things," IEEE Transactions on Green Communications and Networking, vol. 5, no. 3, pp. 1066-1076, 2021.

[20] Y. Zheng, M. D. Alshehri, L. Hai, V. Balasubramanian, M. Zeng, and G. Nie, "Cognitive AmBC-NOMA IoV-MTS networks with IQI: reliability and security analysis," IEEE Transactions on Intelligent Transportation Systems, vol. 53, no. 445 , pp. $232-244,2021$.

[21] S. Tang, "Dilated convolution based CSI feedback compression for massive MIMO systems," IEEE Transactions on Vehicular Technology, 2021.

[22] Z. Zhao, "System optimization of federated learning networks with a constrained latency," IEEE Transactions on Vehicular Technology, vol. 71, 2021.

[23] L. He and K. He, "Towards optimally efficient search with deep learning for large-scale MIMO systems," IEEE Transactions on Communications, pp. 1-12, 2022.

[24] X. Lai, "Outdated access point selection for mobile edge computing with cochannel interference," IEEE Transactions on Vehicular Technology, 2021.

[25] J. Lu, "Analytical offloading design for mobile edge computing based smart internet of vehicle," EURASIP Journal on Advances in Signal Processing, vol. 99, pp. 1-10, 2022.

[26] X. Lai, "Secure mobile edge computing networks in the presence of multiple eavesdroppers," IEEE Transactions on Communications, vol. 70, no. 1, 2021.

[27] L. Fan and X. Lei, "Learning-Based MIMO detection with dynamic spatial modulation," Science China Information Sciences, vol. 99, pp. 1-10, 2022.

[28] K. He and Y. Deng, "Efficient memory-bounded optimal detection for GSM-MIMO systems," IEEE Transactions on Communications, vol. 99, pp. 1-12, 2022.

[29] Y. Guo, S. Lai, H. H. Yang, and T. Q. S. Quek, "Distributed machine learning for multiuser mobile edge computing systems," IEEE Journal of Selected Topics in Signal Processing, vol. 99, 2021.

[30] R. Zhao and M. Tang, "Profit maximization in cache-aided intelligent computing networks," Physical Communication, vol. 99, pp. 1-10, 2022.
[31] S. Tang and L. Chen, "Computational intelligence and deep learning for next-generation edge-enabled industrial IoT," IEEE Transactions on Network Science and Engineering, vol. 99, pp. 1-12, 2022.

[32] L. Zhang, "Dqn based mobile edge computing for smart internet of vehicle," EURASIP Journal on Advances in Signal Processing, vol. 99, pp. 1-10, 2022.

[33] J. Chen and F. ZHou, "Physical-layer security on mobile edge computing for emerging cyber physical systems," IEEE Transactions on Vehicular Technology, vol. 2022, no. 8, pp. 115-126, 2022. 